\title{
Homologous recombination deficiency: New biomarkers in innovative treatments
}

\author{
Christian F. Singer
}

(C) Springer-Verlag GmbH Austria, part of Springer Nature 2020

Homologous recombination (HR) is a key DNA repair mechanism that is essential for maintaining genome stability. It is characterized by its high fidelity and efficiency, and represents the pivotal repair mechanism for DNA double-strand breaks. Although known for years, its potential role as a therapeutic target has only recently been elucidated: malignant breast and ovarian tumors with mutations in the breast cancer susceptibility genes BRCA1 and BRCA2, two genes that are quintessential members of the homologous recombination repair (HRR) pathway, have been demonstrated to be particularly sensitive to polyADP ribose polymerase inhibitors (PARPi), such as talazoparib and olaparib. In addition, BRCA-associated HR deficiency (HRD) renders tumors particularly sensitive to platinum-based chemotherapy.

After the initial approval of PARP inhibitors in advanced ovarian cancer in 2014, their therapeutic indications now span a wide range from BRCAassociated solid tumors from prostate to pancreatic cancers. BRCA testing has thus expanded from a purely predictive to a therapeutic assay. There is, however, mounting evidence suggesting that tumors might benefit from PARPi even in the absence of functional BRCA mutations. Alterations in several other HR-associated genes have been associated with HR deficiency, but the identification of patients with wild-type $B R C A 1 / 2$ who nonetheless will benefit from PARP inhibition remains a significant challenge.

Assays measuring HRD which is caused by a broader range of mechanisms than $B R C A 1 / 2$ loss are now becoming increasingly popular for their ability to iden-

\section{F. Singer, MD, MPH ( $\triangle)$}

Department of Obstetrics and Gynecology, Medical

University of Vienna, Währinger Gürtel 18-20, 1090 Vienna, Austria

christian.singer@meduniwien.ac.at tify an additional subset of PARPi-sensitive. These tests identify both somatic and germline mutations in $B R C A 1 / 2$ and in other HRR-related genes, and detect the presence of genomic alterations indicative of HRD. The so-called genomic "scars" are characterized by telomer-allelic imbalances, large scale transitions and loss of heterocytosity, and-when scored according to a specific algorithm-summarize the total burden of genomic burden in a particular tumor, and describe tumors that are potentially responsive to PARP inhibition.

Several HRD assays have been developed and are now also clinically validated in patients with breast and ovarian cancer, and are now increasingly used in other solid tumors, as well. Recent approvals for the two PARP inhibitors niraparib and olaparib encompass patients with HRD-positive ovarian cancer in addition to those in whom germline or somatic BRCA mutations have been diagnosed. In May 2020, the FDA (US Food and Drug Administration) also approved olaparib for patients with metastatic castration-resistant prostate cancer (mCRPC) with germline or somatic HRR mutations. Until now, the FDA has approved two HRD tests to be used as companion diagnostics: myChoice CDx (Myriad Genetics, Salt Lake City, UT, USA) for niraparib and olaparib in ovarian cancer, and FoundationOne CDx (Foundation Medicine, Cambridge, MA, USA) for olaparib in mCRPC.

While it is now widely accepted that HRD testing allows identification of patients who obtain maximum benefit from PARPi, it is also becoming increasingly evident that some HRD-negative tumors can still respond from the use of PARPi. The reason for this is still unclear and certainly necessitates further investigations. Nevertheless, HR-associated testing and HR-directed therapy will be an essential part of the 


\section{editorial}

pretherapeutic assessment of several solid tumors in the years to come.

The articles presented in this issue of MEMOMagazine of European Medical Oncology are intended to give an overview [1-4].

With kind regards,

\section{Christian Singer}

Conflict of interest C.F. Singer declares that he has no competing interests.

\section{References}

1. Bartl T, Paspalj V, Grimm C. Homologous recombination deficiency in epithelial ovarian cancer. memo. 2020;13(4). https://doi.org/10.1007/s12254-020-00606-z.

2. Marhold M, Topakian T. Homologous repair deficiencies and currentinsights in clinical evaluation of PARPinhibitors in prostate cancer. memo. 2020;13(4). https://doi.org/10. 1007/s12254-020-00599-9.

3. Bartl T, Farr A. Homologous recombination deficiency in breast cancer. memo. 2020;13(4). https://doi.org/10.1007/ s12254-020-00624-x.

4. Doleschal B. Tackling DNA damage repair mechanisms - a promising molecular informed therapeutic approach in pancreatic ductal adenocarcinoma. memo. 2020;13(4). https://doi.org/10.1007/s12254-020-00658-1.

Publisher's Note Springer Nature remains neutral with regard to jurisdictional claims in published maps and institutional affiliations.

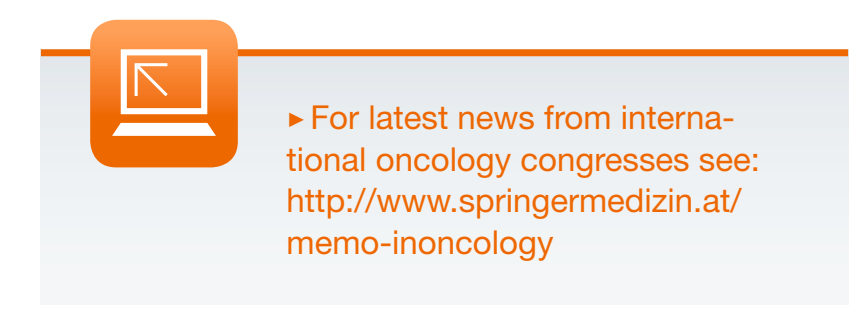

